\title{
Diagnostic Efficacy of Ultrasonography and Fine-needle Aspiration Cytology in Correlation with Histopathology in Euthyroid Patients Having Solitary Thyroid Nodule
}

\author{
Vijay Kumar Sharma, Antony Abraham Paulose, Parvendra Singh, Nishi Sonkhya \\ Department of Otorhinolaryngology and Head and Neck surgery, Sawai Man Singh Medical College, Jaipur, India \\ Email address: \\ drsonkhya ayahoo.co.in (N. Sonkhya), paulose.antony 9 gmail.com (A. A. Paulose)

\section{To cite this article:} \\ Vijay Kumar Sharma, Antony Abraham Paulose, Parvendra Singh, Nishi Sonkhya. Diagnostic Efficacy of Ultrasonography and Fine-needle \\ Aspiration Cytology in Correlation with Histopathology in Euthyroid Patients Having Solitary Thyroid Nodule. Clinical Medicine Research. \\ Vol. 8, No. 1, 2019, pp. 1-5. doi: 10.11648/j.cmr.20190801.11
}

Received: April 26, 2018; Accepted: May 14, 2018; Published: February 25, 2019

\begin{abstract}
Objective: In patients with solitary thyroid nodules, first approach is to confirm whether the nodule is benign or malignant. Commonly available investigations used in the evaluation include thyroid hormone assays, fine needle aspiration cytology (FNAC) and ultrasonography (USG) among others. These procedures are not without drawbacks. The present study was undertaken to compare the diagnostic efficacy of USG and FNAC in correlation with histopathology in euthyroid patients having solitary thyroid nodule. Study Design: A prospective study was carried out on 48 euthyroid cases of solitary thyroid nodule attending the Department of ENT, SMS Medical College \& Hospital, Jaipur, during the period of March 2016 to November 2017. All patients underwent ultrasonography and fine-needle aspiration cytology. The results of FNAC and USG were correlated with post surgical histopathogical examination (HPE) of the specimens to evaluate their sensitivity and specificity by statistical methods. Results: Ultrasound was $87.5 \%$ sensitive \& $92.50 \%$ specific in the detection of malignancy in solitary thyroid nodules while the fine needle aspiration cytology had a sensitivity of $87.50 \% \&$ specificity of $100 \%$ in the same regard. Conclusion: It was found that FNAC is a safe, reliable and cost effective diagnostic modality with a high sensitivity and specificity and is the single best investigation for preoperative evaluation of solitary thyroid nodule to differentiate between benign and malignancy nodules.
\end{abstract}

Keywords: STN, USG, FNAC, Thyroid Malignancy

\section{Introduction}

Solitary thyroid nodule in the general population is very common with an estimated prevalence that ranges from $4 \%$ by palpation to $67 \%$ by ultrasonography. [1,2] Autopsy studies reveal that $50 \%$ of adults had nodules, the majority of which were impalpable. [3] Thyroid nodules are four times more common in females than in men and increase in prevalence with increase in age. [3-5]

Few subjects in surgery have generated as much controversy as the management of solitary thyroid nodule (STN), the two major issues being the diagnostic workup and the extent of thyroidectomy. Because of the possibility of malignancy, some clinicians, especially those in the surgical subspecialties, recommend that all nodules should be removed weighing the risks and price to be paid for a malignancy. [6] On the other hand, endocrinologists recommend that FNAC be performed as the initial step of evaluation in order to avoid unnecessary surgery. Because thyroid nodularity is so common, it would be impossible to operate on every patient with a thyroid mass, as the incidence of malignancy is quite low compared with the overall incidence of thyroid nodularity. $[7,8]$

Thyroid cancer incidence has been increasing over the last two or three decades in high-resource countries in which the disease is currently the second most frequent cancer, after breast cancer, among women younger than 45 years. In highresource countries, papillary and follicular carcinomas, known collectively as differentiated thyroid carcinoma, account for approximately $75 \%$ and $13 \%$ of thyroid cancer, respectively. The well established risk factors for thyroid cancer include prior exposure to ionizing radiation for 
childhood head and neck malignancy, history of goitre and thyroid adenoma. [9] The most common type of thyroid cancer is papillary thyroid cancer (PTC), comprising $80 \%$ of all cases. The second most common type is follicular thyroid cancer (FTC), which accounts for $10 \%$ to $20 \%$ of all cases. [10] Medullary thyroid cancer (MTC), however, arises from the parafollicular $\mathrm{C}$ cells. [11] This neuroendocrine thyroid tumor represents $6 \%$ to $8 \%$ of all thyroid cancer cases and occurs in both familial and sporadic forms. [12] Anaplastic thyroid cancer (ATC) is one of the most aggressive and rapidly fatal cancers. It can develop from DTC on dedifferentiation over time or it also arises de novo. [13]

It has been established from various studies that the majority of thyroid nodules are benign and only $10-20 \%$ of them are malignant. Hence unless indicated, to operate upon every thyroid nodule would be excessive and would relegate a large majority of patients to an unnecessary surgical procedure. So, the goal of diagnostic workup now is to select those patients for surgery who have a high likelihood of harbouring malignancy in the nodule. [14]

In patients with STNs, the first course of action is to determine whether the nodule is benign or malignant. Many investigations are used to differentiate between benign and malignant nodules so as to avoid unnecessary surgical excisions. Among these, FNAC and USG are commonly used in association with clinical features but there are drawbacks for each technique. [5] USG findings are largely subjective and are prone for errors in high-volume institutes. $[15,16]$ Although fine needle aspiration/ biopsy (FNAB) is the most commonly used diagnostic technique for the preoperative evaluation of thyroid nodules, there remains the issue of gray-zone nodules that need further diagnostic investigation. $[14,17]$ The present study was undertaken to compare the diagnostic efficacy of USG and FNAC in correlation with histopathology in euthyroid patients having solitary thyroid nodule.

\section{Materials and Methods}

A prospective study was carried out on 48 euthyroid cases of solitary thyroid nodule attending the Department of ENT, SMS Medical College \& Hospital, India, during the period of March 2016 to November 2017. For the purpose of inclusion in this study, a solitary thyroid nodule (STN) was defined as a single clinically palpable discrete lesion involving either the lobe or the isthmus of the thyroid gland. All patients underwent FNAC, USG and thyroid function test. The results of FNAC were interpreted as benign, malignant, suspicious and inadequate aspirate. The nodules were evaluated for size, location, echotexture, margins, presence of halo, calcification, accessory nodules, associated cervical lymphadenopathy and consistency (solid, cystic or mixed) in order to differentiate between benign and malignant nodules on sonography following which all patients were subjected to surgery and histopathological examination (HPE) of the specimen. The histopathology reports were correlated with the findings of FNAC and USG in order to evaluate their sensitivity and specificity.

\section{Results}

Patient demographics, ultrasonography findings, FNA cytological reports, thyroglobulin and thyrotropin assays are summarized in Table 1. Table 2 shows the distribution of the cases based on ultrasonography findings with $38(79.17 \%)$ cases to be solid on echotexture and rest 10 cases $(20.83 \%)$ cystic. It was observed that out of 48 cases, 10 cases were suspicious for malignancy on USG. Among these 10 suspicious cases, 7 cases were found to be malignant on gold standard HPE and 3 cases were benign on HPE (Table 3 ).

In this study, out of the 48 fine needle aspirates obtained, majority of cases revealed a benign nodule on cytology $(\mathrm{n}=34,70.83 \%)$ followed by malignancy in five aspirates $(10.42 \%)$, follicular lesion of undetermined significance in four cases $(8.33 \%)$, non-diagnostic in three cases $(6.25 \%)$ and suspicious follicular neoplasm in two cases (4.16\%). Table 4 shows the distribution of FNAC findings based on Bethesda classification. Most of the nodules were grade II (70.83\%) on Bethesda classification followed by grade VI $(10.42 \%)$, grade III $(8.33 \%)$, grade I $(6.25 \%)$ and grade IV $(4.16 \%)$ in that order. On comparing with final histopathological diagnosis, FNAC identified seven out of eight malignant cases with an overall accuracy of $97.91 \%$ (Table 6).

Sensitivity and specificity of USG to differentiate malignant from benign thyroid nodule was found to be $87.5 \%$ and $92.50 \%$ respectively with an overall accuracy of $91.67 \%$. The overall sensitivity, specificity, PPV and NPV of FNAC was $87.50 \%, 100 \%, 100 \%$ and $97.50 \%$ (Table 6).

Table 1. Patient Characteristics, USG, FNA and HPE findings.

\begin{tabular}{|c|c|}
\hline Patient Characteristics & Number (\%) \\
\hline Sample Size & 48 \\
\hline Male & $9(18.75 \%)$ \\
\hline Female & $39(81.25 \%)$ \\
\hline $\mathrm{F}: \mathrm{M}$ ratio & $4.3: 1$ \\
\hline Mean Age & $36.08 \pm 13.925$ \\
\hline \multicolumn{2}{|l|}{ USG Findings } \\
\hline Solid & $38(79.17 \%)$ \\
\hline Cystic & $10(20.83 \%)$ \\
\hline \multicolumn{2}{|l|}{ Bethseda Classification } \\
\hline I & $3(6.25 \%)$ \\
\hline II & $34(70.83 \%)$ \\
\hline III & $4(8.33 \%)$ \\
\hline IV & $2(4.16 \%)$ \\
\hline V & $5(10.41 \%)$ \\
\hline \multicolumn{2}{|l|}{ Histopathological Analysis } \\
\hline Benign & $40(83.33 \%)$ \\
\hline Malignant & $8(16.67 \%)$ \\
\hline Incidence Of Malignancy & $16.68 \%$ \\
\hline Male & $22.22 \%$ \\
\hline Female & $15.38 \%$ \\
\hline
\end{tabular}


Table 2. Correlation of USG Echotexture with malignancy.

\begin{tabular}{|c|c|c|c|c|}
\hline \multirow{2}{*}{ Echotexture } & \multirow{2}{*}{$\begin{array}{l}\text { No. of } \\
\text { Cases(\%) }\end{array}$} & \multicolumn{2}{|c|}{ Histopathology } & \multirow{2}{*}{$\begin{array}{l}\text { Percentage of } \\
\text { Malignancy }\end{array}$} \\
\hline & & Benign & Malignant & \\
\hline Cystic & $10(20.83)$ & 10 & 0 & 0 \\
\hline Solid & $38(79.17)$ & 30 & 8 & $21.05 \%$ \\
\hline Total & 48 & 40 & 8 & $16.68 \%$ \\
\hline
\end{tabular}

Table 3. Correlation of USG with Histopathology

\begin{tabular}{lll}
\hline $\begin{array}{l}\text { Suspected malignancy on USG } \\
\text { histological assessment }\end{array}$ & HPR \\
\cline { 2 - 3 } & Malignant & Benign \\
\hline Suspicious for Malignancy (10 cases) & $7(70 \%)$ & $3(30 \%)$ \\
Benign(38cases) & $1(2.63 \%)$ & $37(97.37 \%)$ \\
Total(48 cases) & $8(16.67 \%)$ & $40(83.33 \%)$ \\
\hline
\end{tabular}

Table 4. Bethesda classification of FNAC aspirates.

\begin{tabular}{lll}
\hline Bethesda system & Number & Percentage\% \\
\hline I & 3 & 6.25 \\
II & 34 & 70.83 \\
III & 4 & 8.33 \\
IV & 2 & 4.16 \\
VI & 5 & 10.42 \\
Total & 48 & 100.00 \\
\hline
\end{tabular}

Table 5. Correlation of FNAC findings with HPR

\begin{tabular}{lll}
\hline Suspected malignancy on & On HPE & \\
\cline { 2 - 3 } FNAC & Malignant & Benign \\
\hline Malignant & 7 & 0 \\
Benign & 1 & 40 \\
Total & 8 & 40 \\
\hline
\end{tabular}

Table 6. Diagnostic Test Statistics of USG and FNAC for evaluation of STN.

\begin{tabular}{lll}
\hline Diagnostic Statistics & USG & FNAC \\
\hline Sensitivity & 87.50 & 87.50 \\
Specificity & 92.50 & 100.00 \\
PPV & 70.00 & 100.00 \\
NPV & 97.37 & 97.50 \\
Accuracy & 91.67 & 97.91 \\
\hline
\end{tabular}

\section{Discussion}

National Comprehensive Cancer Network (NCCN) suggests all thyroid nodules be evaluated with thyrotropin and USG of thyroid and neck as a first step and prefers FNA (with or without sono-guidance) as an investigation of choice in only suspicious lesions. [18] Overall incidence of malignancy in STN varies from $9 \%$ to $30 \%$ according to various reports. This study finds an overall incidence of malignancy in STN of $16.67 \%$. USG is a safe and effective method of determining the size and the presence of solid or cystic components within a thyroid nodule. High-resolution USG can be used to determine the presence of nonpalpable nodules as small as $1 \mathrm{~mm}$ within the thyroid tissue. The predictive value of several ultrasonic features of thyroid nodules, including calcifications, margins, and vascularity, have been examined by numerous studies. [19]

Elastosonography in combination with high-resolution USG can significantly improve the diagnostic accuracy to ultrasound which along with molecular markers now available for FNAB to distinguish a malignant from benign thyroid nodules. [20] In a review of published studies, use of conventional thyroid USG did not allow accurate prediction of the histology of STNs. In current practice, its main indications are the accurate measurement of size of the nodule, assessment for possible lymphadenopathy, and for sonoguidance of FNAB. [21] Katz \& Kane et al also found USG to be very accurate in diagnosing adenomatous goitre but found it less reliable to differentiate thyroiditis from a malignant lesion. Out of 21 sonographic suspects, two were later found to be malignant follicular carcinoma. Halo sign was not characteristic of benign follicular adenoma as it was seen in only three out of four $(75 \%)$ cases of follicular adenomas and when halo sign was absent in one case, histopathology revealed follicular adenoma. [22]

In the present series, out of 48 cases, ten cases were found suspicious for malignancy on USG. Among these 10 suspicious cases, seven cases were found to be malignant on gold standard HPE and three cases were benign giving an overall sensitivity and specificity of $87.50 \%$ and $92.50 \%$ respectively for USG to differentiate between benign and malignant nodules. These findings concurs with those of Watters et al who found that the sensitivity, specificity \& positive predictive value of USG in suggesting a malignant disease were $74 \%, 83 \% \& 51 \%$ respectively and they interpreted a solid or mixed solid-cystic and hypoechoic nodule with absence of halo on USG to be suggestive for malignancy. Further, they emphasized that USG has an added advantage in that it allows the whole gland to be examined rather than just the dominant nodule but at the same time, limited by the fact that no features are pathognonomic for malignancy. [23] Hence, USG should be regarded as complementary test rather than an alternative to FNAC for STNs. Jones et al found $75 \%$ sensitivity \& $61 \%$ specificity for USG in suggesting a thyroid nodule to be malignant and they added that whenever there is doubt about the possibility of malignancy, it is safer to operate. [24] Moon and colleagues categorized the internal composition of a nodule according to the ratio of the cystic portion to the solid portion into two groups; predominantly solid $(<50 \%$ cystic) and predominantly cystic ( $\geq 50 \%$ cystic). [25] Kumar et al found $45 \%$ nodules to be solid, $6 \%$ cystic \& $49 \%$ mixed in his series with $14 \%$ of the solid nodules and $9 \%$ of the mixed nodules to be malignant. None of the cystic nodules were malignant in his series. [26] Cox et al reported 6\% incidence of malignancy in cystic nodules \& emphasized that USG of the thyroid is accurate in determining the physical characteristic of isolated swellings but inaccurate in predicting the presence of neoplasia. [27]

Fine needle aspiration cytology appears to be a safe, reliable \& cost effective method which provides valuable information to assist in selection of patients with solitary thyroid nodules for surgery. As it distinguishes the benign from malignant lesions quite effectively preoperatively, it has been proposed as a preoperative screening method of choice. [28]

Fine-needle aspiration biopsy (FNAB) has become the diagnostic tool of choice for the initial evaluation of solitary 
thyroid nodule because of its accuracy, safety, and cost effectiveness. Fewer patients have undergone thyroidectomy for benign disease as a result of FNAB, with resultant decreased health care costs. Although needle biopsy can be performed easily, consistently obtaining adequate tissue and processing the specimens to achieve accurate cytopathological interpretation requires expertise and experience. [29] FNAC specimens are classified as malignant, benign, indeterminate (suspicious for follicular or Hürthle cell neoplasm), or insufficient for diagnosis. The effectiveness of FNAB of solitary thyroid nodules may be improved with the use of ultrasound guidance rather than simple palpation. [30]

Accuracy of FNAB is closely related to the histologic type of thyroid carcinoma that is being evaluated. Papillary thyroid carcinoma is readily identified using FNAB because of its unique cytological features. Diagnosis was correct for papillary thyroid carcinoma in approximately $90-100 \%$ of FNAB specimens when correlated with the histology of the final surgical specimen. Undifferentiated (anaplastic) carcinoma, medullary thyroid carcinoma, and primary thyroid lymphoma also have characteristic cytological features, which aid correct diagnosis in approximately $90 \%$ of FNAB specimens. [31]

In the present study, out of the 48 aspirates obtained, FNAC gave a diagnosis of benign lesion in 34 cases $(70.83 \%)$, followed by malignancy in five cases $(10.42 \%)$, follicular lesion of undetermined significance in four cases $(8.33 \%)$, non-diagnostic in three cases (6.25\%) and suspicious follicular neoplasm in two cases $(4.16 \%)$ which on final histopathological examination found $83.33 \%$ of the thyroid enlargement to be benign $(n=40)$ and rest $16.66 \%$ malignant $(\mathrm{n}=8)$.

Goellner and Gharib reported colloid goitre (40\%) to be the most common histological diagnosis followed by follicular adenoma (18\%) and adenomatous goitre in about $11 \%$ cases with a $16 \%$ malignancy rate. [32] This study found $16.67 \%$ cases $(n=8)$ to be malignant on histological examination of which seven were rightly diagnosed positive for malignancy on FNAC. The overall accuracy was $97.91 \%$ in our series [Table 6], compared to $79 \%$ to $98 \%$ accuracy in other series.

A study by Kaliszewski et al indicated that FNAB rarely produces false-negative results in patients with solitary malignant thyroid tumors, while in contrast, the results in patients with multiple malignant thyroid tumors are often false negative. Compared with the thyroid cancer prediction rate for FNAB in patients with multiple malignant nodules, the prediction rate for those with cancer in single nodules was three times higher. [33] In this series, FNAC gave false negative diagnosis in one patient without any false positive cases.

Since the present study has included small number of cases ( $n=48)$, comparison with other studies is showing the difference of interpretation results. Accuracy rate of FNAC depends greatly on who prepares the cytology smears and how it is interpreted. Other possible reason for the misinterpretation could be the occurrence of hyperplastic nodules in the gland. The only way of reducing this would be to take more aspirates from solid areas. Cystic changes in STNs are a common cause for missed or wrong diagnosis. Altavila and Pascale reported in their series a $45.83 \%$ concordance between the histological \& cytological diagnosis of papillary carcinoma. On including the suspect cases as cytologically positive, the concordance with histology was 70\%. [34]

This study finds an overall sensitivity of $87.50 \%$ and specificity of $100 \%$ for FNAC in diagnosing a malignancy in a solitary thyroid nodule.

\section{Conclusion}

To conclude, even while chances of malignancy in a solitary thyroid nodule are less, keeping in mind the higher prevalence for STNs, it would be pragmatic to diagnose the malignant potential of STNs rather than a close observation protocol. Combining USG with FNAC proves to be a costeffective method for screening thyroid malignancy in economically backward geographical areas till affordable and more definitive alternatives arise.

\section{References}

[1] Ezzat S, Sarti DA, Cain DR. Braunstein GD. Thyroid incidentalomas, prevalence by palpation and ultrasonography. Arch Intern Med 1994; 154: 1838-40.

[2] Hegedus L. Clinical practice. The thyroid nodule. $N$ Engl $J$ Med 2004; 351: 1764-1771.

[3] Vander JB, Gaston EA, Dauber TR. The significance of nontoxic thyroid nodules. Final report of 15 years study of incidence of thyroid malignancy. Ann Intern Med 1968; 53740 .

[4] Mazzaferri EL, de los Santos ET, Rofagha-Keyhani S. Solitary thyroid nodule: diagnosis and management. Med Clin North Am 1988; 72: 1177-1211.

[5] Cooper DS, Doherty GM, Haugen BR, et al. Revised American Thyroid Association management guidelines for patients with thyroid nodules and differentiated thyroid cancer. Thyroid 2009; 19:1167-1214.

[6] Cole WH, Majarakis JD. Incidence of carcinoma of the thyroid in nodular goitre. J Clin Endocrinol. 1949;9:1007-11

[7] Layfield LJ, Cibas ES, Gharib H, Mandel SJ. Thyroid aspiration cytology: current status. CA Cancer $J$ Clin 2009;59:99-110.

[8] Thompson NW, Nishiyama RH, Harness JK. Thyroid carcinoma: Current controversies. Curr Probl Surg 1978;15:1.

[9] Rinaldi S, Plummer M, Biessy C, et al, 2014 Thyroid stimulating hormone, thyroglobulin, and thyroid hormones and risk of differentiated thyroid carcinoma: the EPIC study. $J$ Natl Cancer Inst 106: Doi: 10.1093/ jnci/dju097

[10] Hedinger C, Williams ED, Sobin H. Histological typing of thyroid tumours: WHO international classification of tumours, $4^{\text {th }}$ edn. Berlin: Springer-Verlag, 1998. 
[11] Williams ED. Histogenesis of medullary carcinoma of the thyroid. Journal of Clinical Pathology 1966;19: 114-18.

[12] Pacini F, Castagna MG, Cipri C, Schlumberger M. Medullary thyroid carcinoma. Clinical Oncology 2010; 22:475-85.

[13] Schneider DF, Chen H. New developments in the diagnosis and treatment of thyroid cancer. CA: a cancer journal for clinicians. 2013;63(6):373-394.

[14] Gharib H, Papini E. Thyroid nodules: clinical importance, assessment, and treatment. Endocrinol Metab Clin North Am. 2007;36(3):707-35.

[15] Czerwonka L, Freeman J, McIver B, et al. Summary of proceedings of the second World Congress on Thyroid Cancer. Head Neck 2014;36:917-920.

[16] Koike E, Noguchi S, Yamashita H, et al. Ultrasonographic characteristics of thyroid nodules: prediction of malignancy. Arch Surg 2001;136:334-337.

[17] Shrestha M, Crothers BA, Burch HB. The impact of thyroid nodule size on the risk of malignancy and accuracy of fineneedle aspiration: a 10 -year study from a single institution. Thyroid 2012;22:1251-1256.

[18] NCCN Clinical Practice Guidelines in Oncology- Thyroid Carcinoma. Version 2.2017-May17, 2017. NCCN.org

[19] Can AS, Peker K. Comparison of palpation-versus ultrasoundguided fine-needle aspiration biopsies in the evaluation of thyroid nodules. BMC Res Notes. 2008 May 15. 1:12.

[20] Goldfarb M, Gondek S, Solorzano C, Lew JI. Surgeonperformed ultrasound can predict benignity in thyroid nodules. Surgery. 2011 Sep. 150(3):436-41.

[21] Hegde A, Gopinathan A, Abu Bakar R, Ooi CC, Koh YY, Lo RH. A method in the madness in ultrasound evaluation of thyroid nodules. Singapore Med J. 2012 Nov. 53(11):766-72.

[22] Katz J. F, Kane R. A. Thyroid nodules: sonographic pathologic correlation. Radiology.1984: Vol.151; 741-745.

[23] Watters DA, Ahuja AT, Evans RM, Chick W, King WW,
Metreweli C, Li AK. Role of ultrasound in the management of thyroid nodules. Am J Surg. 1992 Dec; 164(6):654-7..

[24] Jones AJ, Altman TJ. Comparison of FNAC, RNS \& USG in the management of thyroid nodules. Post Grad Med $J$ 1990: $66 ; 914-17$.

[25] Moon WJ, Jung SL, Lee JH, Na DG, Baek JH, Lee YH, et al. Benign and malignant thyroid nodules: US differentiation multicenter retrospective study. Radiology. 2008; 247:762-70.

[26] ArunKumar, Ahuja MMS et al FNAC, USG \& RNS in STN. $J$ Assoc Physicians India 1992:40; 302 -306

[27] Cox MR Marshall SG STN A prospective scanning \& USG Br. J Surg 1991 78, 90-93.

[28] Basharat R, Bukhari MH, Saeed S, Hamid T. Comparison of fine needle aspiration cytology and thyroid scan in solitary thyroid nodule. Patholog Res Int. 2011. 2011:754041.

[29] Yip L, Farris C, Kabaker AS, Hodak SP, Nikiforova MN, McCoy KL, et al. Cost impact of molecular testing for indeterminate thyroid nodule fine-needle aspiration biopsies. J Clin Endocrinol Metab. 2012 Jun. 97(6):1905-12.

[30] Arul P, Masilamani S. A correlative study of solitary thyroid nodules using the Bethesda System for Reporting Thyroid Cytopathology. J Cancer Res Ther. 2015 Jul-Sep. 11 (3):61722.

[31] Bakkar S, Poma AM, Corsini C, Miccoli M, Ambrosini CE, Miccoli P. Underestimated risk of cancer in solitary thyroid nodules $\geq 3 \mathrm{~cm}$ reported as benign. Langenbecks Arch Surg. 2017 Jul 8.

[32] Goellner JR, Gharib H. FNAC Of the thyroid. ActaCytologica. 1987: 31; 587-591.

[33] Kaliszewski K, Diakowska D, Wojtczak B, et al. Fine-Needle Aspiration Biopsy as a Preoperative Procedure in Patients with Malignancy in Solitary and Multiple Thyroid Nodules. PLoS One. 2016. 11 (1):e0146883.

[34] Altavilla G, Pascale M. FNAC of thyroid gland diseases. ActaCytologica 1990 34, 251-256. 